\title{
Dendron-polymer hybrids as tailorable coronae of single-walled carbon nanotubes
}

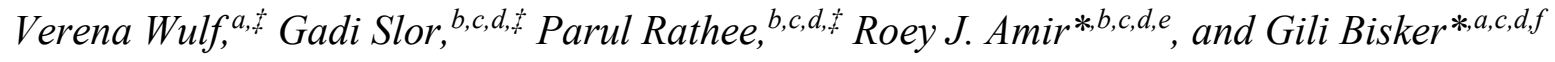

${ }^{a}$ Department of Biomedical Engineering, Faculty of Engineering, Tel-Aviv University, Tel Aviv 6997801, Israel

${ }^{b}$ Department of Organic Chemistry, School of Chemistry, Faculty of Exact Sciences, Tel-Aviv University, Tel-Aviv 6997801, Israel

${ }^{\mathrm{c}}$ The Center for Physics and Chemistry of Living Systems, Tel-Aviv University, Tel Aviv 6997801, Israel

${ }^{\mathrm{d}}$ Center for Nanoscience and Nanotechnology, Tel-Aviv University, Tel Aviv 6997801, Israel ${ }^{\mathrm{e} A D A M A}$ Center for Novel Delivery Systems in Crop Protection, Tel-Aviv University, Tel Aviv 6997801, Israel

${ }^{f}$ Center for Light Matter Interaction, Tel-Aviv University, Tel Aviv 6997801, Israel

*Email: amirroey@tauex.tau.ac.il; bisker@tauex.tau.ac.il 


\begin{abstract}
Single-walled carbon nanotubes (SWCNTs), non-covalently functionalized by synthetic polymers, find widespread applications including sensing and imaging. Identifying new amphiphiles with interchangeable building blocks that can form unique coronae around the SWCNT, customized for a specific application, is thus of a great interest. We present polymerdendron hybrids, composed of hydrophobic dendrons and hydrophilic polyethylene glycol (PEG), as amphiphilic macromolecules with high degree of structural freedom, for suspending SWCNTs in aqueous solution. Based on a set of four PEG-dendrons differing in their dendritic end-groups, we show that differences in the chemical structure of the hydrophobic end-groups control the interactions of the PEG-dendrons with the SWCNT-surface. These interactions led to differences in the intrinsic near-infrared fluorescence emission of the SWCNTs and affected the PEG-dendron susceptibility to enzymatic degradation, which was monitored by the SWCNT fluorescent signal. Our findings open new avenues for rational design of SWCNT functionalization, and optical sensing of enzymatic activity.
\end{abstract}




\section{Introduction}

Single-walled carbon nanotubes (SWCNTs) find widespread application as biomedical sensors, mainly due to their intrinsic fluorescence emission in the near infrared transparency window of biological tissue. ${ }^{1-7}$ SWCNTs can be described as graphene sheets rolled up into cylinders, resulting in nanotubes with different $(n, m)$-chiralities, dependent on their roll-up vector. ${ }^{8}$ The fluorescence emission peak intensities and wavelengths of a SWCNT-sample are dependent on its composition of nanotubes with different chiralities and the dielectric environment. ${ }^{9-11}$

Owing to their graphene-like surface, SWCNTs are highly hydrophobic nanostructures that require functionalization with dispersants such as low molecular weight surfactants, ${ }^{12}$ singlestranded DNA, ${ }^{13}$ RNA, ${ }^{14}$ suitable proteins, ${ }^{15}$ or amphiphilic polymers, ${ }^{16,17}$ in order to form colloidal suspensions in aqueous media. The interactions enabling a dispersant to bind the SWCNT-surface are primarily $\pi-\pi$-stacking between the graphene lattice and an aromatic group (DNA, polymers) ${ }^{13}$, or hydrophobic interactions (surfactants, phospholipids). ${ }^{15}$

For sensing applications, these dispersing agents either contain a recognition element specific for the desired analyte (e.g., aptamers, antibodies, binding peptides),${ }^{18-23}$ or they wrap the SWCNTs forming a so-called corona phase, that due to its structure, conformation, and charge is able to selectively bind certain analytes. ${ }^{24-26}$ Upon the interaction with the target analyte, the emitted fluorescence of the SWCNTs is modulated. Corona-based recognition was reported for different types of analytes, including small biomolecules, ${ }^{25,27}$ and even proteins. ${ }^{28-30}$ The importance of the nature of the exposed, hydrophilic part of the dispersant in forming an analytespecific corona phase is evident, but previous publications reveal that the corona phase is also influenced by its hydrophobic anchor unit, attached to the SWCNTs. For the recognition of fibrinogen, SWCNTs suspended by a polyethylene glycol (PEG) polymer coupled to a 
phospholipid with a chain length of 16 carbon atoms were found to have the highest optical response, while coupling to a phospholipid with 14 or 18 carbon atoms showed significantly weaker response. ${ }^{28}$ This hints to the fact that the polymer, which is exposed to the solution and thus to the analyte, as well as the hydrophobic anchor of the amphiphile both play equally important roles in the formation of the corona phase.

Recently, several approaches have emerged for producing libraries of amphiphilic linear copolymers and block-copolymers that can be used for suspending SWCNT for specific applications ${ }^{31}$. These include combinatorial high-throughput screening of a library of polymers, ${ }^{25,27,28}$ high-throughput directed evolution, ${ }^{32,33}$ reversible addition-fragmentation chain transfer polymerization, ${ }^{34,35}$ and random peptide synthesis. ${ }^{36,37}$ Nevertheless, challenges such as elaborated synthesis, multiple processing steps, or limited control over the final product, give rise to the need for novel approaches for generating a library of highly versatile amphiphilic polymers with a wide range of molecular structures and functions.

Dendrons are tree-like molecular structures with multiple end-groups, depending on the number of branching points, i.e. the dendron generation, and a reactive centre that enables coupling, e.g., to a polymer. Dendrons can vary in flexibility, size, and hydrophobicity, providing high degrees of freedom and molecular precision in creating hydrophobic structures. Combining hydrophobic dendron bearing hydrophobic end-groups with hydrophilic polymers, yields amphiphilic polymerdendron hybrids. ${ }^{38,39}$ The stability of micellar assemblies of these amphiphilic macromolecules, in particular PEG-dendron hybrids, have been widely studied and found to be dependent on the amphiphilic ratio between the polymer tail and the hydrophobic dendron. ${ }^{40,41}$ Dendrons can be coupled to a variety of polymers to create a library of amphiphilic molecules with the ability to suspend SWCNTs in aqueous environment and providing designable wrapping agents to address 
the requirements of a particular application. ${ }^{42}$ Previously, polystyrene coupled to pyrenefunctionalized dendrons were reported to suspend SWCNTs in THF, ${ }^{43}$ while hydrophilic dendrons coupled to a hydrophobic alkyl-chain or pyrene have been reported to suspend SWCNTs in water, due to increasing hydrophilicity with increasing dendron generation. ${ }^{44}-47$ Nevertheless, designing hydrophobic dendrons as the binding sites provides a high degree of control over their direct interaction with the SWCNT surface. ${ }^{48}$

In this study, we present polymer-dendron hybrids as modular amphiphilic agents for the suspension of SWCNTs in aqueous media. The polymer-dendron hybrids designed for this study are amphiphilic macromolecules consisting of three building blocks (Fig. 1A): I) A hydrophilic polymer chain, whose hydrophilicity and flexibility depends on its chemical composition and its length, II) The dendron body acting as a hydrophobic block, whose size, flexibility and hydrophobicity are determined by the chemical structure of the branching units and the dendron's generation, III) A number of hydrophobic end-groups depending on the generation of the dendron. To shed light on the effect of the dendritic end-groups on the corona phase around the SWCNTs, and the resulting optical properties, we designed PEG-dendron amphiphiles with end-groups that can differ in their interactions with the carbon nanotube surface. Taking advantage of the high modularity and simplicity of the synthetic pathway, we studied the influence of precise changes in the chemical structure of the end-groups on the fluorescence emission of the SWCNTs. Taking enzymatic cleavage of the dendron end-groups as a model, we also tested the reactivity of the PEGdendrons corona of the SWCNTs, which can be monitored via fluorescence spectroscopy in the nIR spectral region, to further understand and exploit the nature of the specific interactions between the anchoring end-groups and the SWCNTs surface. 


\section{Results and Discussion}

The dendron-polymer hybrids, applied in this study, contain mono-methoxy polyethylene glycol as the hydrophilic polymer chain. PEG was proven to be a suitable polymer for the dispersion of SWCNTs, providing a hydrophilic shell for their stable suspensions in water and biological media and being relatively inert towards protein interaction. ${ }^{28,49,50}$ The dendritic architecture exposes four hydrophobic end-groups, enabling non-covalent binding to the SWCNTs. To create a set of polymer-dendrons, two aliphatic and two aromatic end-groups were coupled via an ester group in two different orientations to the dendritic body (Fig. 1B), resulting in PEG-D-naphthyl, PEG-Dnaphthoate, PEG-D-pentyl, and PEG-D-hexanoate (Scheme S1). The orientation of the ester group in the end-groups places either an electron acceptor (carbonyl group of the ester) for PEG-Dnaphthoate, or an electron donor (alkoxy group of the ester) for PEG-D-naphthyl, in vicinity of the delocalized $\pi$-electron system of the aromatic end-group affecting its electron density. Due to their aromatic nature, these end-groups should bind to the SWCNTs via $\pi$ - $\pi$-stacking interactions, which are expected to be influenced by the electron density of the delocalized $\pi$-systems. ${ }^{51}$ In contrast, the aliphatic end-groups interact with the SWCNTs via hydrophobic interactions. Hence, the orientation of the ester group is not expected to have a significant effect on the binding interaction. To avoid an effect due to changes in molecular weight, each couple of amphiphiles, was synthesized to have the same molecular weight. This was achieved by using 2-mercaptoethanol for preparing the 2-naphthoate and hexanoate end-groups, while 3-mercaptopropionic acid was reacted with the 2-naphthol and pentanol for synthesizing the naphthyl and pentyl end-groups, respectively (Scheme S4). 

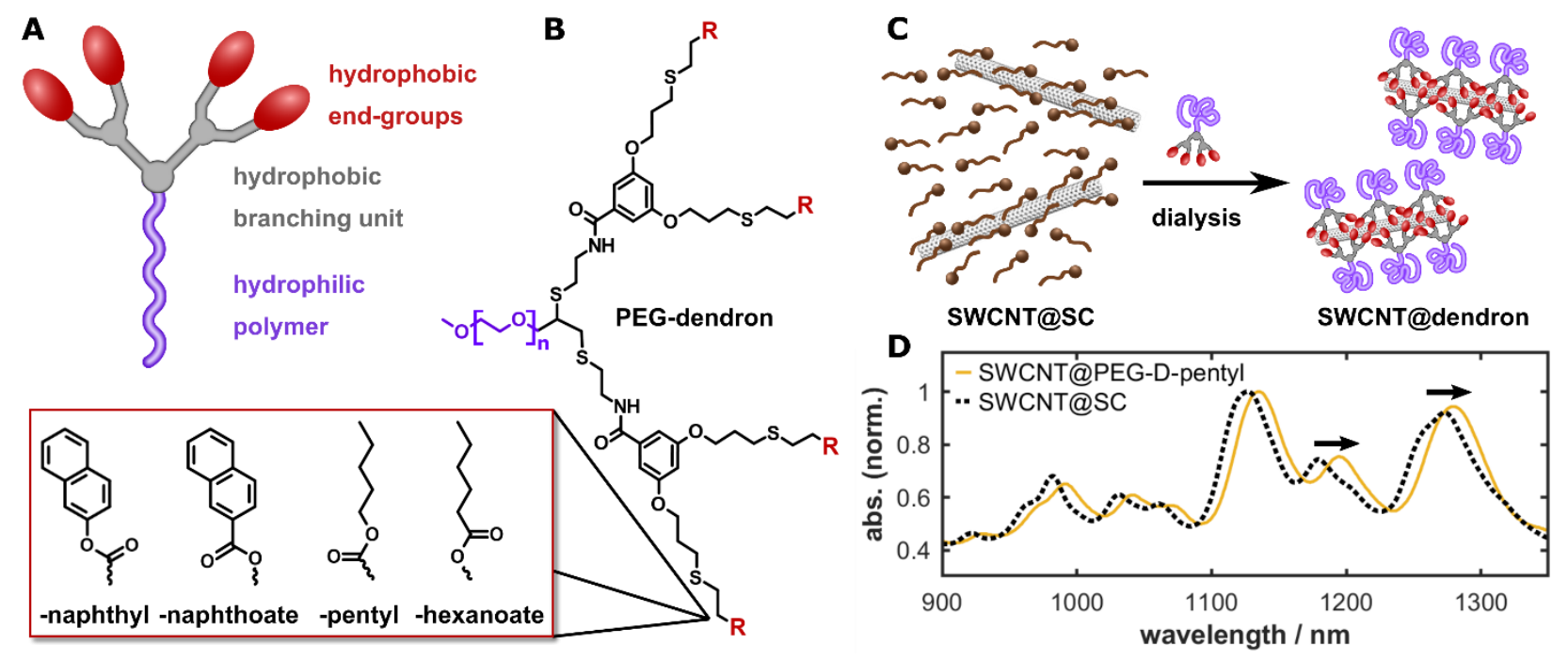

Figure 1. Polymer-dendron hybrids are modular amphiphilic macromolecules capable to disperse SWCNTs. A) Schematic representation and chemical structure of the amphiphilic polymerdendron hybrids consisting of a hydrophilic polymer chain (purple), and a hydrophobic dendron (grey) that bears hydrophobic end-groups (red). (B) Polyethylene glycol (PEG, $5 \mathrm{kDa}$ ) serves as the hydrophilic chain, coupled to a dendron branching into four identical hydrophobic end-groups. The hydrophobic end-groups, naphthyl, naphthoate, pentyl and hexanoate, differ in their aromaticity (naphthalene-based or aliphatic) and in the orientation of an ester group. C) Functionalization of SWCNTs with the polymer-dendron amphiphiles (SWCNT@PEG-dendrons) was performed via surfactant exchange from sodium cholate suspended SWCNTs (SWCNT@SC). SWCNT@SC together with PEG-dendrons are dialyzed against water to slowly remove sodium cholate and enable attaching of PEG-dendrons. D) Absorption spectra of the SWCNT@PEGdendrons, here shown for SWCNT@PEG-D-pentyl (yellow solid line), compared to the initial absorption of the SWCNT@SC (black dashed line), show a red shift, which indicates a change in the dielectric environment of the SWCNTs, due to the binding of the PEG-dendron amphiphiles.

Functionalization of the SWCNTs was achieved via surfactant exchange, where sodium cholate suspended SWCNT (SWCNT@SC), together with the respective polymer-dendron, were dialyzed 
against water, to slowly remove sodium cholate allowing the PEG-dendrons to bind the SWCNTs and stabilize them in suspension (Fig. 1C). ${ }^{28}$ Performing the surfactant exchange with a PEGdendron bearing hydrophilic end-groups resulted in SWCNTs aggregation (Supplementary Fig.11), showing that the hydrophobic end-groups are essential for stabilizing the suspended SWCNTs. The nIR-absorption spectra of the resulting PEG-dendron wrapped SWCNT

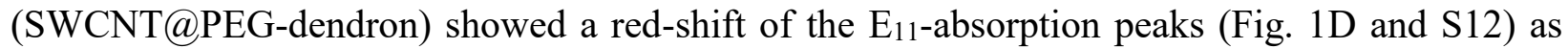
well as of the fluorescence emission of the SWCNT chiralities (Supplementary Fig.13) compared to SWCNT@SC, indicating a successful wrapping exchange by confirming a change in the dielectric environment of the SWCNTs. ${ }^{52}$
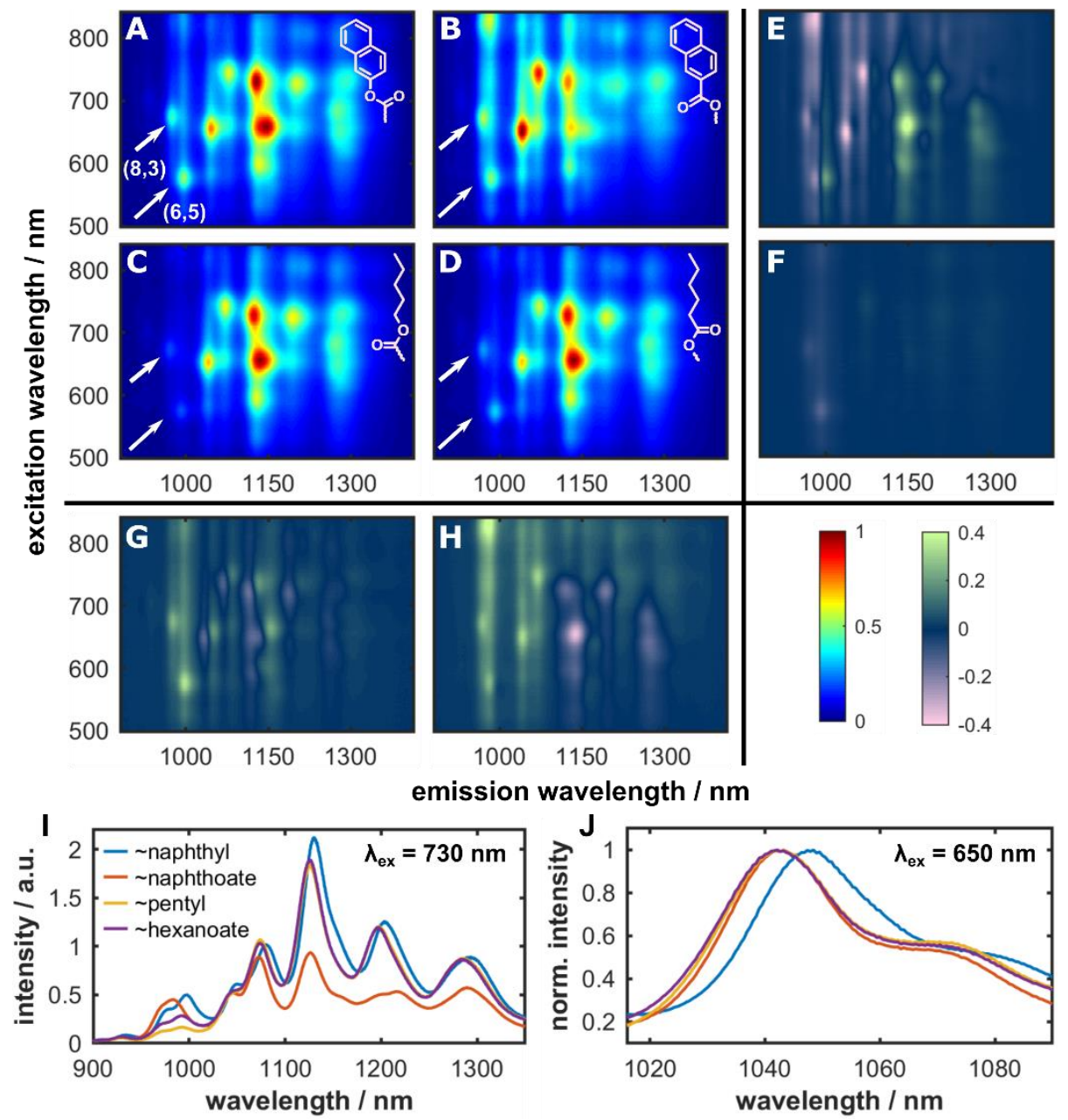
Figure 2. Fluorescence emission of SWCNT@PEG-dendrons reveal chirality-dependent fluorescence emission for the different end-groups. Normalized fluorescence excitation-emission spectra for A) SWCNT@PEG-D-naphthyl, B) SWCNT@PEG-D-naphthoate, C) SWCNT@PEG-D-pentyl, and D) SWCNT@PEG-D-hexanoate, each at a concentration of $5 \mathrm{mg} / \mathrm{L} . \mathrm{E}-\mathrm{H})$ Differences between the two respective neighboring normalized fluorescence plots. E) Difference in fluorescence emission of the aromatic end-groups (A and B). F) Difference in fluorescence emission of the aliphatic end-groups (C and D). G and H) Difference in fluorescence emission of the aromatic end-groups vs. its aliphatic counterpart having the same orientation of the ester group (A and C; B and D). I) Differences in fluorescence emission intensity at $\lambda_{\mathrm{ex}}=730 \mathrm{~nm}$ for SWCNT@PEG-D-naphthyl (blue), SWCNT@PEG-D-naphthoate (red), SWCNT@PEG-D-pentyl (yellow), SWCNT@PEG-D-hexanoate (purple) at a concentration of 5 mg/L. SWCNT@PEG-D-naphthoate has significantly lower fluorescence emission intensity except for $(8,3)$-and $(6,5)$-chiralities. J) normalized fluorescence emission for the $(7,5)$ chirality at an excitation wavelength of $\lambda_{\mathrm{ex}}=650 \mathrm{~nm}$ reveals shifts in the emission wavelength (SWCNT@PEG-D-naphthyl (blue), SWCNT@PEG-D-naphthoate (red), SWCNT@PEG-Dpentyl (yellow), SWCNT@PEG-D-hexanoate (purple)).

Each corona phase induces, due to its interactions or density, a distinct dielectric environment around the SWCNTs that influences their fluorescence emission. ${ }^{53}$ Our PEG-dendrons are bound to the SWCNTs via end-groups differing in the type of their ability to interact with the graphene lattice. While $\pi$ - $\pi$-stacking of the SWCNT with the aromatic end-groups is possible, hydrophobic interactions are expected in the case of the aliphatic end-groups. Differences in these interactions are reflected in different fluorescence emission spectra of the SWCNT@PEG-dendron samples. While the four SWCNT@PEG-dendron show comparable absorption spectra of the $\mathrm{E}_{22}$-transitions 
(Supplementary Fig.15), we, indeed, observe substantial differences in their fluorescence emission spectra of the $\mathrm{E}_{11}$-transitions, although all four samples were prepared from the same initial SWCNT@SC suspension. Fig.s 2A-D show the nIR-fluorescence emission recorded for excitation wavelengths of $\lambda_{\mathrm{ex}}=500-840 \mathrm{~nm}$ for the four different SWCNT@PEG-dendrons while Fig.s 2E$\mathrm{H}$ show the differences in the normalized fluorescence emission between the two respective neighbouring plots. The orientation of the ester-group in case of the two PEG-dendrons with aromatic end-groups, and the resulting difference of their electron density on $\pi$ - $\pi$-stacking interactions had a dramatic effect on SWCNT fluorescence modulation (Fig. 2A, 2B, and 2E). However, for the two PEG-dendrons with aliphatic end-groups we observed comparable fluorescence emission, since the orientation of the ester-group has a minimal effect on the hydrophobic interactions (Fig. 2C, 2D, and 2F). A clear impact on fluorescence emission can also be observed when comparing the aliphatic end-groups with their aromatic counterparts having the ester group in the same orientation (Fig. 2G-H). Most evident is the high fluorescence emission of the small-diameter $(8,3)$ and $(6,5)$ chiralities wrapped by the aromatic end-groups compared to the aliphatic ones. These variations cannot be attributed to simple differences in the concentration of the chiralities within the samples, as evident from the similar absorption spectra (Supplementary Fig.15), but rather to the distinct chemical properties of the end-groups. We clearly see that differences in the nature of the interaction between the amphiphilic wrapping and the SWCNTs result in differences in the fluorescence intensity (Fig. 2I) and shifts in fluorescence emission as shown for the $(7,5)$ chirality (Fig. 2J). 

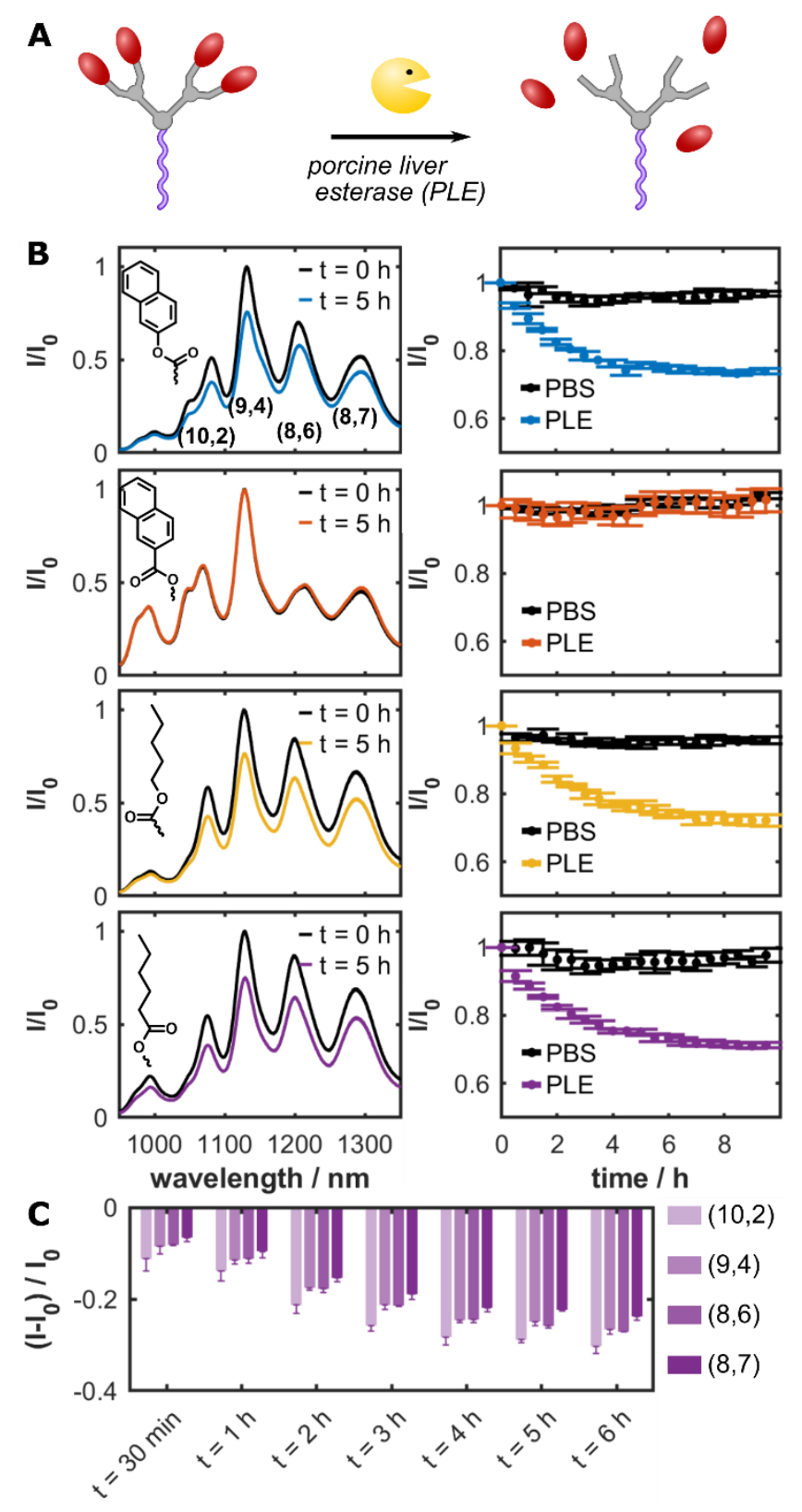

Figure 3. Fluorescence response of SWCNT@PEG-dendrons to porcine liver esterase (PLE). A) Cleavage of the PEG-dendrons by PLE takes place at the ester group connecting the end-groups to the dendron body. B) Fluorescence response of SWCNT@PEG-D-naphthyl (blue), SWCNT@PEG-D-naphthoate (red), SWCNT@PEG-D-pentyl (yellow), and SWCNT@PEG-Dhexanoate (purple) each at a SWCNT-concentration of $1 \mathrm{mg} / \mathrm{L}$ upon incubation with PLE $1.5 \mu \mathrm{M}$ for $5 \mathrm{~h}(\lambda \mathrm{ex}=730 \mathrm{~nm})$ and time dependent fluorescence response after the addition of PLE of the 
peak intensity of the $(9,4)$ chirality at $\lambda \mathrm{ex}=730 \mathrm{~nm}$ over $10 \mathrm{~h}$ compared to a sample in PBS (black dots). C) Time dependent fluorescence response of four peaks of the different chiralities, in resonance with the excitation wavelength at $\lambda \mathrm{ex}=730 \mathrm{~nm}$, for SWCNT@PEG-D-hexanoate upon the addition of PLE: $(10,2)$ at $\lambda \mathrm{em} \sim 1075 \mathrm{~nm},(9,4)$ at $\lambda \mathrm{em} \sim 1130 \mathrm{~nm},(8,6)$ at $\lambda \mathrm{em} \sim 1200 \mathrm{~nm}$, $(8,7)$ at $\lambda \mathrm{em} \sim 1285 \mathrm{~nm}$. The chiralities show comparable decay rate but different overall fluorescence change, which is correlated to the diameter $d$ of the chiralities $(\mathrm{d}(10,2)=0.87 \mathrm{~nm}$; $\mathrm{d}(9,4)=0.90 \mathrm{~nm} ; \mathrm{d}(8,6)=0.95 \mathrm{~nm} ; \mathrm{d}(8,7)=1.02 \mathrm{~nm})$.

Specific fluorescence emission of the SWCNT@PEG-dendrons not only reveals different interaction between the SWCNT and PEG-dendrons, but also implies that the noncovalent binding stability of the PEG-dendrons depends on their end-groups. Amphiphilic PEG-dendrons are known to self-assemble into polymeric micelles, whose stability is dependent on their hydrophilic/hydrophobic ratio. ${ }^{54-56}$ The ester group connecting the hydrophobic end-groups to the dendritic branches is susceptible to enzymatic cleavage by porcine liver esterase (PLE), which can cleave off naphthol, naphthoic acid, pentanol, and hexanoic acid end-groups (Fig. 3A). Enzymatic cleavage should yield carboxylic acid or alcohol end-groups on the dendritic block, decreasing its hydrophobicity, and, thus, resulting in PEG-dendrons with a substantially higher hydrophilic/hydrophobic ratio. ${ }^{55,57}$ The overall degradation rate of the PEG-dendrons in micellar assemblies was shown to depend on the equilibrium between 'monomeric' PEG-dendrons and their micellar assemblies. ${ }^{58}$ Translated to the SWCNT@PEG-dendrons, we expected their enzymatic degradation to depend on the non-covalent binding interactions between the nanotubes and the hydrophobic end-groups of the PEG-dendrons. Changes in the corona phase, by detaching and/or cleavage of the wrapping amphiphiles, should induce changes in the dielectric environment of the nanotubes, which can be monitored via their nIR-fluorescence emission ${ }^{10,28,53,59}$. In order to 
follow the PEG-dendron degradation, we incubated the SWCNT@PEG-dendrons to PLE, and monitored the fluorescence response during an incubation time of $10 \mathrm{~h}$. Significant fluorescence modulation could be observed for SWCNT@PEG-D-naphthyl, SWCNT@PEG-D-pentyl, and SWCNT@PEG-D-hexanoate, showing a decrease in intensity of ca. 30\%, while SWCNT@PEGD-naphthoate showed no response (Fig. 3B left). The extent of the decrease in fluorescence intensity reflects the differences in SWCNT/PEG-dendron interactions mentioned above. The orientation of the ester-bond in the aromatic end-group significantly influenced the fluorescence response, as a result of the different electron density of the aromatic group. However, comparing the response of the aliphatic end-groups showed no significant differences between the two orientations. The absorption spectra of SWCNT@PEG-dendrons before and after incubation with PLE for $24 \mathrm{~h}$ showed no changes in absorption confirming that the fluorescence intensity decrease cannot be explained by simple aggregation of the SWCNT (Supplementary Fig.16). Unspecific protein interaction with the SWCNTs was excluded as incubating the SWCNT@PEG-dendrons with bovine serum albumin (BSA), a protein commonly used for assays of non-specific binding, showed no fluorescence response (Supplementary Fig.17). Monitoring the time-dependent fluorescence decrease of the $(9,4)$ chirality for the different SWCNT@PEG-dendrons showed comparable decay rates for SWCNT@PEG-D-naphthyl, SWCNT@PEG-D-pentyl and SWCNT@PEG-D-hexanoate and saturation after 4-5 h (Fig. 3B right). Quantifying the fluorescence response of the different chiralities in each of the samples when excited at $\lambda_{\mathrm{ex}}=730 \mathrm{~nm}$, showed a correlation between the normalized fluorescence response and the nanotube-diameter, where smaller diameter SWCNTs showed larger response (Fig. 3C, S18 and Table S2). Diameter dependent effects of SWCNTs on the interactions with adsorbed molecules can be attributed to differences in available surface area or SWCNT-curvature. ${ }^{60-62}$ 


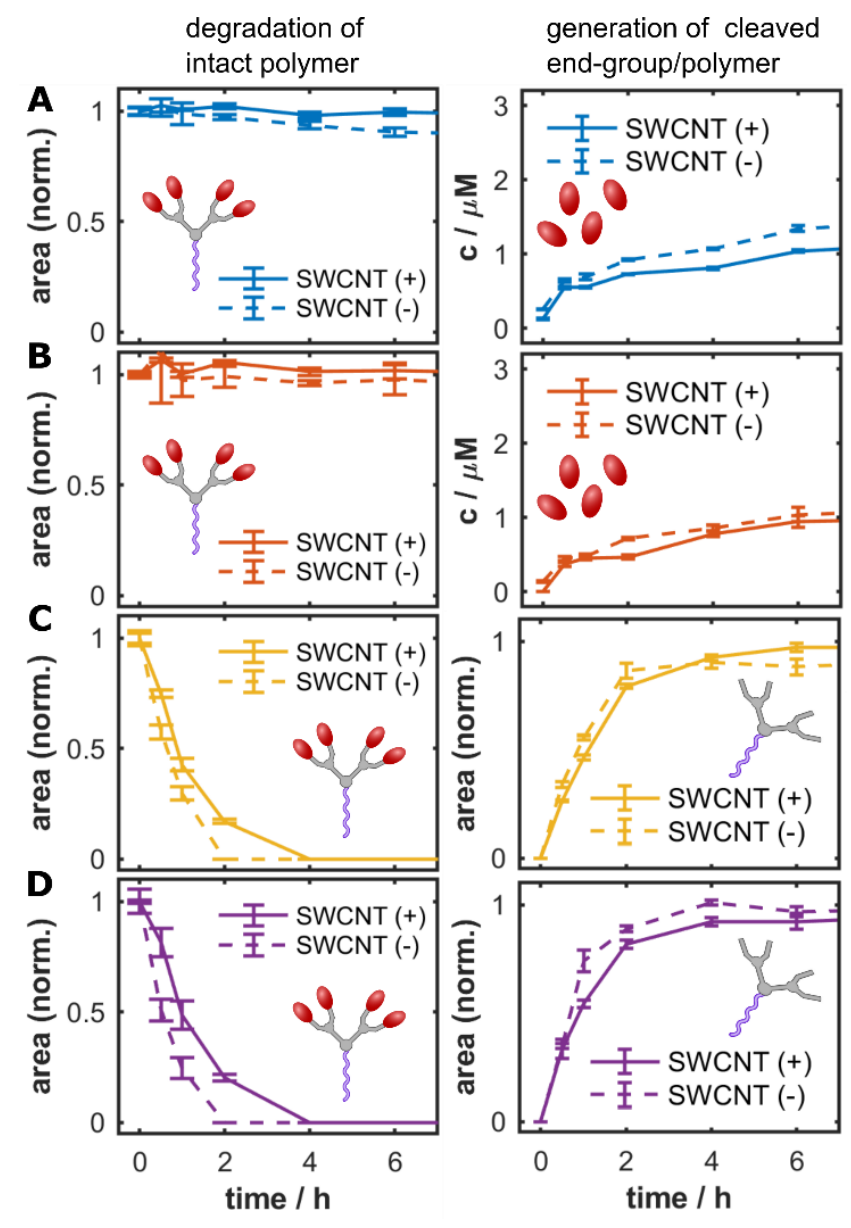

Figure 4. Quantification of the degradation process of PEG-dendrons wrapping SWCNTs and as free micellar assemblies after separation via HPLC. Enzymatic degradation process at time intervals of $(0 ; 0.5 ; 1 ; 2 ; 4 ; 6$ h). A) PEG-D-naphthyl (blue); B) PEG-D-naphthoate (red); C) PEGD-pentyl (yellow): D) PEG-D-hexanoate (purple) as free micellar assemblies in absence of SWCNT (SWCNT(-); dashed lines) and as wrapping polymer around SWCNTs (SWCNT(+); solid lines). Left column: Degradation of the intact polymer, bearing all four end-groups. Right Column: Quantification of the concentration of the cleaved-off end-groups naphthol (blue) and naphthoic acid (red). Generation of polymer-dendrons with cleaved-off end-groups for PEG-Dpentyl (yellow) and PEG-D-hexanoate (purple). 
In order to gain a better understanding of the mechanism governing the fluorescence response to the interaction with the esterase, we used high performance liquid chromatography (HPLC) to directly follow and quantify the enzymatic degradation of the amphiphiles (Supplementary Fig.2021). To this end, SWCNT@PEG-dendrons were incubated with PLE at room temperature for 6 hours in total. During the incubation, fractions of the samples were extracted for further testing. The extracted samples were mixed with acetonitrile in 1:1 volume ratio in order to quench the enzyme activity and precipitate the SWCNT, while keeping the PEG-dendrons in solution. The same procedure was repeated for a comparable concentration of the PEG-dendrons in micellar assemblies in solution $(50 \mu \mathrm{g} / \mathrm{mL})$ without the nanotubes (Supplementary Fig.19 and Table S3). Taking advantage of the ability to quantify the reaction components by HPLC, we could directly follow the rate of degradation of the intact PEG-dendrons, i.e., PEG-dendrons with all four endgroups (Fig. 4 A-D left). In addition, owing to the spectroscopic signal of naphthol and naphthoic acid, which is lacking in the case of pentanol and hexanoic acid, we could also follow the increasing concentration of the cleaved-off end-groups of PEG-D-naphthyl and PEG-Dnaphthoate (Fig. 4 A-B right). In case of PEG-D-pentanol and PEG-D-hexanoate we could monitor the generation of the cleaved polymer (Fig. 4 C-D right). For all four PEG-dendrons, the degradation process of the intact PEG-dendrons was comparable for SWCNT@PEG-dendrons (Fig. 4, solid lines) and the 'free' PEG-dendrons (Fig. 4, dashed lines), but slightly faster for the latter. The aromatic PEG-dendrons showed a very slow, and incomplete degradation process, as the samples still contained $>95 \%$ of the intact PEG-dendrons after $6 \mathrm{~h}$ incubation time (Fig. 4A), in agreement with the concentration of the cleaved-off aromatic end-groups over time (Fig. 4B and calibration curve Supplementary Fig.22). Following the degradation of the intact aliphatic PEGdendrons as well as the generation of their cleaved PEG-dendrons without end-groups, we monitor 
full enzymatic degradation after an incubation time of ca. $4 \mathrm{~h}$. The slow degradation rate for the aromatic PEG-dendrons shows that their micellar assemblies, as well as their binding to the SWCNTs surface, are much more stable. This higher stability renders them substantially less susceptible to enzymatic degradation compared to the aliphatic ones.

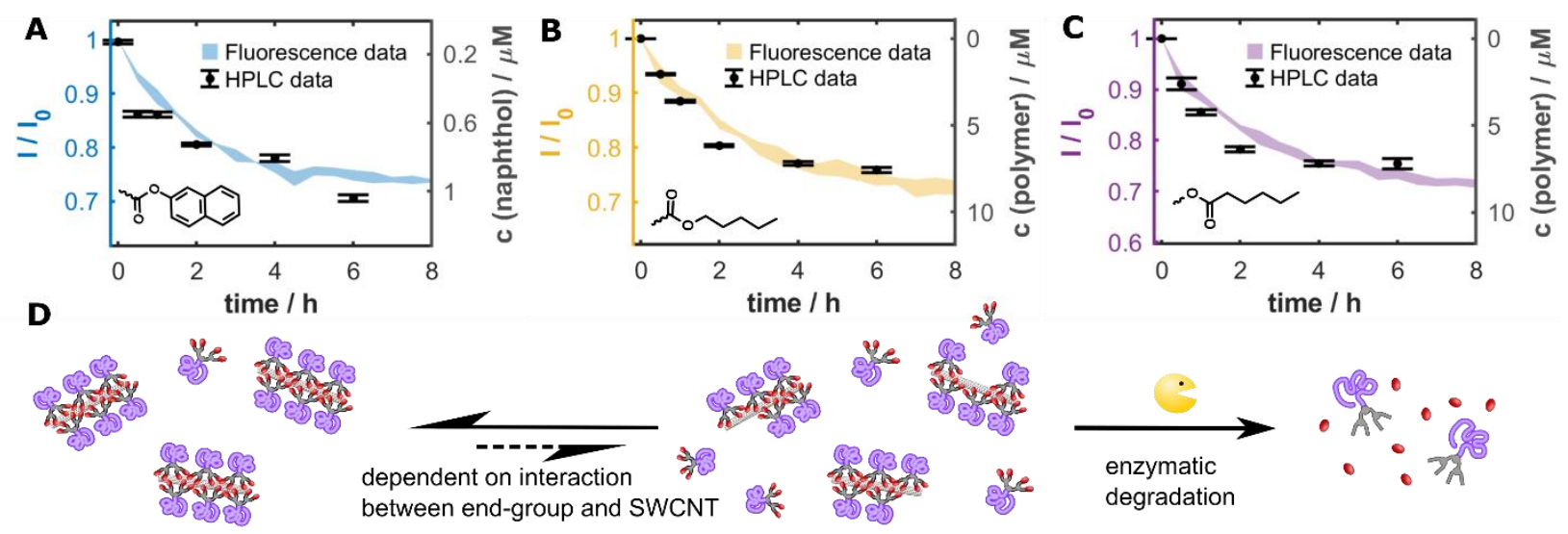

Figure 5. Correlation of the fluorescence signal to the degradation of the polymer-dendrons and proposed mechanism. A) Correlation of the time dependent fluorescence signal of SWCNT@PEG-D-naphthyl (blue) to the concentration increase of the cleaved-off end-group naphthol determined via HPLC (black; $\mathrm{R}=-0.95, \mathrm{P}=0.003$ ). $\mathrm{B}$ and $\mathrm{C}$ ) Correlation of the time dependent fluorescence signal of SWCNT@PEG-D-pentyl (yellow; R=-0.98, P=0.001) and SWCNT@PEG-D-hexanoate (purple; R=-0.97, P=0.001) to the generation of the cleaved polymer, measured after separation via HPLC (black). Shaded areas show mean and standard deviation. D) Proposed equilibrium dependent degradation mechanism, which is dependent on the interaction between SWCNT/PEG-dendron assemblies.

The fluorescence modulation of the SWCNT as a function of time, measured by the nIR emission of the SWCNT@PEG-dendrons, can be correlated to the generation of cleaved naphthol endgroups in case of PEG-D-naphthyl or to the generation of cleaved polymers from PEG-D-pentyl and PEG-D-hexanoate (Fig. 5A-C). Surprisingly, although no spectral response was noted for 
SWCNT@PEG-D-naphthoate, the amount of cleaved end-groups were comparable to the case of SWCNT@PEG-D-naphthyl which showed a decrease of the fluorescence intensity. (Fig. 3B and 4B). The results obtained after the separation of the reaction components via HPLC cannot distinguish between PEG-dendrons that were bound directly to the SWCNT surface or PEGdendrons that are in solution, while the fluorescence signal solely monitors events in close proximity to the SWCNT surface. We therefor assume that in case of PEG-D-naphthoate most of the cleaved-off end-groups stem from PEG-dendron molecules that are not directly bound to the SWCNTs, thus, there is no effect on the fluorescence emission of the SWCNTs. The proposed mechanism for the enzymatic degradation of PEG-dendrons wrapping SWCNTs is depicted in Fig. 5D. Non-covalent binding of PEG-dendrons to the SWCNTs depends on the interactions of the respective end-groups with the SWCNT-surface. While aliphatic end-groups undergo full degradation as was tracked by HPLC, and could also be monitored via their fluorescence emission, the aromatic end-groups led to assemblies with the SWCNTs that are much more stable but differed based on their specific $\pi$ - $\pi$-stacking. Naphthoate end-groups form assemblies that are not susceptible to enzymatic degradation, while naphthyl end-groups can be removed from the SWCNT surface up to a certain extent.

\section{Conclusion}

We herein showed a family of amphiphilic PEG-dendrons that are able to disperse SWCNTs in water through direct interactions between their hydrophobic end-groups, serving as molecular binding sites, and the graphene lattice of the SWCNTs. Using enzymatic degradation of the PEGdendrons by esterase activity as a model, differences in the stability and reactivity of these assemblies were shown to be related to differences in the intrinsic fluorescence emission of the SWCNT@PEG-dendron samples. The two aliphatic PEG-dendrons are attached to the SWCNTs 
by simple hydrophobic interactions and show similar fluorescence emission as well as similar stability and reactivity. However, due to the ester group acting either as an electron acceptor or an electron donor towards the naphthalene $\pi$-system, we observe big differences in fluorescence modulation between these end-groups, including fluorescence emission intensity and wavelength. The differences in the electron density of the delocalized $\pi$-system also results in different susceptibility of the assemblies towards enzymatic cleavage. In contrast to SWCNT@PEG-Dnaphthyl, the fluorescence emission of SWCNT@PEG-D-naphthoate does not reveal changes in the corona upon addition of the esterase.

The amphiphilic polymer-dendron hybrids introduced here are tailorable macromolecules synthesized with high molecular precision, whose structure and composition can be easily tuned simply by changing the hydrophobic end-groups, branching unit, and hydrophilic polymer. The dendron body can be designed to have branches with varying flexibility and generation number, resulting in different numbers of end-groups and interaction sites on the SWCNT-surface. The polymer chain can vary in charge, flexibility, conductivity, or can even be designed to attach recognition elements or functional groups. The versatile polymer-dendron structures open endless possibilities to design of enzyme-responsive polymeric functionalization of nIR fluorescent SWCNT. This new platform of configurable functional composite nanomaterials offers the opportunity to tailor it for numerous applications studying stability, reactivity, and susceptibility to enzymatic activity in real-time through the transient modulation of the fluorescence emission in the nIR spectral window. 


\section{Methods}

Synthesis of PEG-dendron amphiphiles. The PEG-dendron amphiphiles were synthesized in a two-step synthesis starting from mono-methoxy $5 \mathrm{kDa}$ polyethylene glycol diamine (mPEG $\mathrm{kDa}^{-}$ $\left(\mathrm{NH}_{2}\right)_{2}$. The two terminal amines were reacted with bis-allyl functionalized $\mathrm{AB}_{2}$ branching units forming stable amide bonds. In the second step the designated cleavable hydrophobic end-groups were coupled to the polymer using photo-initiated thiol-ene click reaction. All amphiphiles were obtained in high yields and were characterized using ${ }^{1} \mathrm{H}-\mathrm{NMR}$, GPC and MALDI-TOF. For additional synthetic information and spectroscopic data please see supporting information.

Suspension of SWCNTs with sodium cholate. To suspend the SWCNTs with sodium cholate, $10 \mathrm{mg}$ of HiPCO SWCNTs in $20 \mathrm{~mL}$ of $2 \%$ (w/v) sodium cholate were bath sonicated for $10 \mathrm{~min}$ and subsequently tip sonicated for $2 \times 30$ min on ice $(12 \mathrm{~W})$. To remove nanotube bundles and impurities, the resulting suspension was ultracentrifuged for $4 \mathrm{~h}$ at 40,000 g (Optima XPN-80 ultracentrifuge, Beckman Coulter). The concentration of SWCNT@SC was determined spectroscopically $\quad(\mathrm{c}(\mathrm{SWCNT} @ \mathrm{SC})=220 \mathrm{mg} / \mathrm{L})$ with an extinction coefficient of $\varepsilon_{632 \mathrm{~nm}}=0.036 \mathrm{~L} \cdot \mathrm{mg}^{-1} \cdot \mathrm{cm}^{-1}$.

Surfactant exchange of SWCNTs with PEG-dendrons. Surfactant exchange was performed via dialysis from SWCNT@SC as described previously. ${ }^{28}$ Briefly, a suspension of SWCNT@SC $(40 \mathrm{mg} / \mathrm{L})$ in $2 \%(\mathrm{w} / \mathrm{v})$ sodium cholate and the respective PEG-dendron $(2 \mathrm{mg} / \mathrm{mL})$ in a total volume of $7 \mathrm{~mL}$ were dialyzed (GeBAflex Mega, $3.5 \mathrm{kDa} \mathrm{MWCO}$ ) against water for 5 days, with daily water exchange. After the dialysis, SWCNT@PEG-dendron were centrifuged for 30 min at $20,000 \mathrm{~g}$ to remove aggregates. The supernatant suspension was washed 3 times with PBS buffer (pH 7.4) in an Amicon centrifugal filter (100 kDa MWCO, $25 \mathrm{~min}, 4,400 \mathrm{~g})$. The concentration of SWCNT@PEG-Dendron suspensions was determined as described above. ${ }^{28}$ 
Absorption spectroscopy. Absorption spectra were recorded in a UV-vis-nIR spectrophotometer (Shimadzu UV-3600 Plus) in a wavelength range of 200-1400 nm.

NIR-fluorescence spectroscopy of carbon nanotubes. Fluorescence emission spectra were recorded in a 96-well-plate mounted on an inverted microscope (Olympus IX73). A supercontinuum white-light laser (NKT-photonics, Super-K Extreme) with a bandwidth filter (NKTphotonics, Super-K varia, $\Delta \lambda=20 \mathrm{~nm}$ ) was coupled into the microscope as the excitation source. If not stated otherwise, spectra were recorded at an excitation wavelength of $\lambda_{e x}=730 \mathrm{~nm}$ with $8.5 \mathrm{~mW}$. Fluorescence emission was spectrally resolved using a spectrograph (Spectra Pro HRS300, Princeton Instruments) with a slit-width of $500 \mu \mathrm{m}$ and a grating $(150 \mathrm{~g} / \mathrm{mm})$. The fluorescence intensity spectrum was recorded by an InGaAs-camera (PylonIR, Teledyne Princeton Instruments) with $1 \mathrm{~s}$ exposure time for $\mathrm{c}(\mathrm{SWCNT})=5 \mathrm{mg} / \mathrm{L}$ or $5 \mathrm{~s}$ for $\mathrm{c}(\mathrm{SWCNT})=1 \mathrm{mg} / \mathrm{L}$. Excitation-emission maps were recorded using an excitation wavelength range of $500 \mathrm{~nm}$ to $840 \mathrm{~nm}$ in $2 \mathrm{~nm}$ steps.

Enzymatic degradation of PEG-dendrons wrapping SWCNTs. Aliquots of $147 \mu \mathrm{L}$ SWCNT@PEG-dendrons in PBS (1 mg/L SWCNT) were placed in a 96 well plate, and treated with $3 \mu \mathrm{L}$ PLE to a final enzyme concentration of $1.5 \mu \mathrm{M}$ or $3 \mu \mathrm{L}$ of PBS as a control for $10 \mathrm{~h}$ incubation time. Fluorescence emission of the samples was measured in time intervals of $30 \mathrm{~min}$. at an excitation wavelength of $\lambda_{\mathrm{ex}}=730 \mathrm{~nm}$.

Separation and quantification of the reaction components of the degradation process via

HPLC. HPLC measurements were recorded on a Waters Alliance e2695 separations module equipped with a Waters 2998 photodiode array detector. A total volume of $10 \mathrm{~mL}$ containing SWCNT@PEG-dendrons $(\mathrm{c}(\mathrm{SWCNT})=1 \mathrm{mg} / \mathrm{L})$ and PLE $(1.5 \mu \mathrm{M})$ and a control containing only PEG-dendrons $(50 \mu \mathrm{g} / \mathrm{mL})$ and PLE $(1.5 \mu \mathrm{M})$ were incubated. At time intervals of $0 \mathrm{~h}, 30 \mathrm{~min}$, 
$1 \mathrm{~h}, 2 \mathrm{~h}, 4 \mathrm{~h}$, and $6 \mathrm{~h}$, a volume of $1 \mathrm{~mL}$ was taken from the samples and subjected to $1 \mathrm{~mL}$ of acetonitrile to precipitate the SWCNTs while keeping the PEG-dendrons and the cleavage products in suspension. The solution was filtered with a $0.2 \mu \mathrm{m}$ PTFE syringe filter to remove SWCNTs. $60 \mu \mathrm{L}$ of the samples were injected into HPLC. For the quantification of the reaction components we integrated the peaks assigned to the intact polymer-dendrons (bearing all 4 end-groups) at absorption wavelength of: $\lambda=225 \mathrm{~nm}$ (PEG-D-naphthyl); $\lambda=236 \mathrm{~nm}$ (PEG-D-naphthoate); and $\lambda=297 \mathrm{~nm}$ (PEG-D-pentyl and PEG-D-hexanoate). In the case of PEG-D-naphthyl and PEG-Dnaphthoate we integrated the peaks assigned to the cleaved-off end-groups, naphthol and naphthoic acid at absorption wavelength of: $\lambda=225 \mathrm{~nm}$ and $\lambda=236 \mathrm{~nm}$, respectively. The concentrations of the cleaved-off end-groups were determined via a calibration curve, determined by the area of the peak for naphthol $(\lambda=225 \mathrm{~nm})$ and naphthoic acid $(\lambda=236 \mathrm{~nm})$ at concentrations of 1,5 , and $10 \mu \mathrm{M}$. In the case of PEG-D-pentyl and PEG-D-hexanoate we integrated the peaks assigned to the cleaved polymer at an absorption wavelength of: $\lambda=297 \mathrm{~nm}$. 
1. Jain, A., Homayoun, A., Bannister, C. W. \& Yum, K. Single-walled carbon nanotubes as near-infrared optical biosensors for life sciences and biomedicine. Biotechnol. J. 10, 447459 (2015).

2. Mann, F. A., Lv, Z., Großhans, J., Opazo, F. \& Kruss, S. Nanobody-Conjugated Nanotubes for Targeted Near-Infrared In Vivo Imaging and Sensing. Angew. Chemie Int. Ed. 58, 11469-11473 (2019).

3. Welsher, K., Sherlock, S. P. \& Dai, H. Deep-tissue anatomical imaging of mice using carbon nanotube fluorophores in the second near-infrared window. Proc. Natl. Acad. Sci. 108, 8943 LP - 8948 (2011).

4. Iverson, N. M. et al. Quantitative tissue spectroscopy of near infrared fluorescent nanosensor implants. J. Biomed. Nanotechnol. 12, 1035-1047 (2016).

5. Godin, A. G. et al. Single-nanotube tracking reveals the nanoscale organization of the extracellular space in the live brain. Nat. Nanotechnol. 12, 238-243 (2017).

6. Beyene, A. G. et al. Imaging striatal dopamine release using a nongenetically encoded near infrared fluorescent catecholamine nanosensor. Sci. Adv. 5, eaaw3108 (2019).

7. Hofferber, E. M., Stapleton, J. A. \& Iverson, N. M. Review—Single Walled Carbon Nanotubes as Optical Sensors for Biological Applications. J. Electrochem. Soc. 167, $037530(2020)$.

8. Saito, R. et al. Optical absorption of graphite and single-wall carbon nanotubes. Appl. Phys. A 78, 1099-1105 (2004).

9. Bachilo, S. M. et al. Structure-assigned optical spectra of single-walled carbon nanotubes. Science (80-. ). 298, 2361-2366 (2002).

10. Gao, J., Gomulya, W. \& Loi, M. A. Effect of medium dielectric constant on the physical 
properties of single-walled carbon nanotubes. Chem. Phys. 413, 35-38 (2013).

11. Campo, J. et al. Optical Property Tuning of Single-Wall Carbon Nanotubes by Endohedral Encapsulation of a Wide Variety of Dielectric Molecules. ACS Nano (2020) doi:10.1021/acsnano.0c08352.

12. Jain, R. M., Ben-Naim, M., Landry, M. P. \& Strano, M. S. Competitive Binding in Mixed Surfactant Systems for Single-Walled Carbon Nanotube Separation. J. Phys. Chem. C 119, 22737-22745 (2015).

13. Zheng, M. et al. DNA-assisted dispersion and separation of carbon nanotubes. Nat. Mater. 2, 338-342 (2003).

14. Landry, M. P. et al. Comparative Dynamics and Sequence Dependence of DNA and RNA Binding to Single Walled Carbon Nanotubes. J. Phys. Chem. C 119, 10048-10058 (2015).

15. Antonucci, A., Kupis-Rozmysłowicz, J. \& Boghossian, A. A. Noncovalent Protein and Peptide Functionalization of Single-Walled Carbon Nanotubes for Biodelivery and Optical Sensing Applications. ACS Appl. Mater. Interfaces 9, 11321-11331 (2017).

16. Fernandes, R. M. F., Dai, J., Regev, O., Marques, E. F. \& Furó, I. Block Copolymers as Dispersants for Single-Walled Carbon Nanotubes: Modes of Surface Attachment and Role of Block Polydispersity. Langmuir 34, 13672-13679 (2018).

17. Budhathoki-Uprety, J. et al. Polymer cloaking modulates the carbon nanotube protein corona and delivery into cancer cells. J. Mater. Chem. B 5, 6637-6644 (2017).

18. Nißler, R. et al. Remote near infrared identification of pathogens with multiplexed nanosensors. Nat. Commun. 11, 5995 (2020).

19. Landry, M. P. et al. Single-molecule detection of protein efflux from microorganisms using fluorescent single-walled carbon nanotube sensor arrays. Nat. Nanotechnol. 12, 
368-377 (2017).

20. Williams, R. M., Lee, C. \& Heller, D. A. A Fluorescent Carbon Nanotube Sensor Detects the Metastatic Prostate Cancer Biomarker uPA. ACS Sensors 3, 1838-1845 (2018).

21. Nelson, J. T. et al. Mechanism of Immobilized Protein A Binding to Immunoglobulin G on Nanosensor Array Surfaces. Anal. Chem. 87, 8186-8193 (2015).

22. Chio, L. et al. Electrostatic Assemblies of Single-Walled Carbon Nanotubes and Sequence-Tunable Peptoid Polymers Detect a Lectin Protein and Its Target Sugars. Nano Lett. 19, 7563-7572 (2019).

23. Shumeiko, V., Paltiel, Y., Bisker, G., Hayouka, Z. \& Shoseyov, O. A paper-based nearinfrared optical biosensor for quantitative detection of protease activity using peptideencapsulated swcnts. Sensors (Switzerland) 20, 1-14 (2020).

24. Zuo, F. et al. Redox-responsive inclusion complexation between beta-cyclodextrin and ferrocene-functionalized poly(N-isopropylacrylamide) and its effect on the solution properties of this polymer. Supramol. Chem. 20, 559-564 (2008).

25. Zhang, J. et al. Molecular recognition using corona phase complexes made of synthetic polymers adsorbed on carbon nanotubes. Nat. Nanotechnol. 8, 959-968 (2013).

26. Hendler-Neumark, A. \& Bisker, G. Fluorescent single-walled carbon nanotubes for protein detection. Sensors 19, (2019).

27. Kruss, S. et al. Neurotransmitter Detection Using Corona Phase Molecular Recognition on Fluorescent Single-Walled Carbon Nanotube Sensors. J. Am. Chem. Soc. 136, 713-724 (2014).

28. Bisker, G. et al. Protein-targeted corona phase molecular recognition. Nat. Commun. 7, (2016). 
29. Bisker, G. et al. Insulin Detection Using a Corona Phase Molecular Recognition Site on Single-Walled Carbon Nanotubes. ACS Sensors 3, 367-377 (2018).

30. Ehrlich, R., Hendler-Neumark, A., Wulf, V., Amir, D. \& Bisker, G. Optical Nanosensors for Real-time Feedback on Insulin Secretion by $\beta$-Cells. bioRxiv 2021.03.21.435763 (2021) doi:10.1101/2021.03.21.435763.

31. Gillen, A. J. \& Boghossian, A. A. Non-covalent Methods of Engineering Optical Sensors Based on Single-Walled Carbon Nanotubes. Front. Chem. 7, 612 (2019).

32. Lambert, B., Gillen, A. J., Schuergers, N., Wu, S.-J. \& Boghossian, A. A. Directed evolution of the optoelectronic properties of synthetic nanomaterials. Chem. Commun. 55, 3239-3242 (2019).

33. Jeong, S. et al. High-throughput evolution of near-infrared serotonin nanosensors. Sci. $A d v .5$, eaay3771 (2019).

34. Lee, M. A. et al. Implantable Nanosensors for Human Steroid Hormone Sensing In Vivo Using a Self-Templating Corona Phase Molecular Recognition. Adv. Healthc. Mater. 9, 2000429 (2020).

35. Dong, J. et al. A synthetic mimic of phosphodiesterase type 5 based on corona phase molecular recognition of single-walled carbon nanotubes. Proc. Natl. Acad. Sci. 117, 26616 LP - 26625 (2020).

36. Shumeiko, V. et al. A nanoscale optical biosensor based on peptide encapsulated SWCNTs for detection of acetic acid in the gaseous phase. Sensors Actuators, B Chem. 327, 128832 (2021).

37. Shumeiko, V., Paltiel, Y., Bisker, G., Hayouka, Z. \& Shoseyov, O. A nanoscale paperbased near-infrared optical nose (NIRON). Biosens. Bioelectron. 172, 112763 (2021). 
38. Gitsov, I., Wooley, K. L. \& Fréchet, J. M. J. Novel Polyether Copolymers Consisting of Linear and Dendritic Blocks. Angew. Chemie Int. Ed. English 31, 1200-1202 (1992).

39. Gitsov, I., Wooley, K. L., Hawker, C. J., Ivanova, P. T. \& Fréchet, J. M. J. Synthesis and properties of novel linear-dendritic block copolymers. Reactivity of dendritic macromolecules toward linear polymers. Macromolecules 26, 5621-5627 (1993).

40. Gitsov, I. Hybrid linear dendritic macromolecules: From synthesis to applications. J. Polym. Sci. Part A Polym. Chem. 46, 5295-5314 (2008).

41. Whitton, G. \& Gillies, E. R. Functional aqueous assemblies of linear-dendron hybrids. $J$. Polym. Sci. Part A Polym. Chem. 53, 148-172 (2015).

42. Slor, G. et al. Judging Enzyme-Responsive Micelles by Their Covers: Direct Comparison of Dendritic Amphiphiles with Different Hydrophilic Blocks. Biomacromolecules 22, 1197-1210 (2021).

43. Bahun, G. J. \& Adronov, A. Interactions of carbon nanotubes with pyrene-functionalized linear-dendritic hybrid polymers. J. Polym. Sci. Part A Polym. Chem. 48, 1016-1028 (2010).

44. Setaro, A. et al. Polyglycerol-derived amphiphiles for single walled carbon nanotube suspension. Chem. Phys. Lett. 493, 147-150 (2010).

45. Ernst, F., Heek, T., Setaro, A., Haag, R. \& Reich, S. Functional surfactants for carbon nanotubes: Effects of design. J. Phys. Chem. C 117, 1157-1162 (2013).

46. Ernst, F. et al. Noncovalent Stable Functionalization Makes Carbon Nanotubes Hydrophilic and Biocompatible. J. Phys. Chem. C 121, 18887-18891 (2017).

47. Gutierrez-Ulloa, C. E. et al. Carbon Nanotubes Decorated with Cationic Carbosilane Dendrons and Their Hybrids with Nucleic Acids. ChemNanoMat 4, 220-230 (2018). 
48. Maeda, Y. et al. Control of near infrared photoluminescence properties of single-walled carbon nanotubes by functionalization with dendrons. Nanoscale 10, 23012-23017 (2018).

49. Iverson, N. M. et al. In vivo biosensing via tissue-localizable near-infrared-fluorescent single-walled carbon nanotubes. Nat. Nanotechnol. 8, 873-880 (2013).

50. Pinals, R. L. et al. Rapid SARS-CoV-2 Detection by Carbon Nanotube-Based NearInfrared Nanosensors. medRxiv 2020.11.02.20223404 (2020) doi:10.1101/2020.11.02.20223404.

51. Poenitzsch, V. Z. et al. Effect of Electron-Donating and Electron-Withdrawing Groups on Peptide/Single-Walled Carbon Nanotube Interactions. J. Am. Chem. Soc. 129, 14724$14732(2007)$.

52. Welsher, K. et al. A route to brightly fluorescent carbon nanotubes for near-infrared imaging in mice. Nat. Nanotechnol. 4, 773-780 (2009).

53. Hertel, T. et al. Spectroscopy of Single- and Double-Wall Carbon Nanotubes in Different Environments. Nano Lett. 5, 511-514 (2005).

54. Harnoy, A. J. et al. Modular Synthetic Approach for Adjusting the Disassembly Rates of Enzyme-Responsive Polymeric Micelles. Biomacromolecules 18, 1218-1228 (2017).

55. Slor, G., Papo, N., Hananel, U. \& Amir, R. J. Tuning the molecular weight of polymeric amphiphiles as a tool to access micelles with a wide range of enzymatic degradation rates. Chem. Commun. 54, 6875-6878 (2018).

56. Segal, M. et al. Molecular Precision and Enzymatic Degradation: From Readily to Undegradable Polymeric Micelles by Minor Structural Changes. J. Am. Chem. Soc. 139, 803-810 (2017). 
57. Harnoy, A. J. et al. Enzyme-responsive amphiphilic PEG-dendron hybrids and their assembly into smart micellar nanocarriers. J. Am. Chem. Soc. 136, 7531-7534 (2014).

58. Slor, G. \& Amir, R. J. Using High Molecular Precision to Study Enzymatically Induced Disassembly of Polymeric Nanocarriers: Direct Enzymatic Activation or EquilibriumBased Degradation? (2021) doi:10.1021/acs.macromol.0c02263.

59. Heller, D. A. et al. Peptide secondary structure modulates single-walled carbon nanotube fluorescence as a chaperone sensor for nitroaromatics. Proc. Natl. Acad. Sci. 108, 8544 LP -8549 (2011).

60. Wu, Q. Y., Tian, W. De \& Ma, Y. Q. Nanopatterns of Phospholipid Assemblies on Carbon Nanotubes: A Molecular Dynamics Simulation Study. J. Phys. Chem. C 122, 7455-7463 (2018).

61. Yurekli, K., Mitchell, C. A. \& Krishnamoorti, R. Small-angle neutron scattering from surfactant-assisted aqueous dispersions of carbon nanotubes. J. Am. Chem. Soc. 126, 9902-9903 (2004).

62. Salem, D. P. et al. Chirality dependent corona phase molecular recognition of DNAwrapped carbon nanotubes. Carbon N. Y. 97, 147-153 (2016). 


\section{Acknowledgements}

G.B. acknowledges the support of the Zuckerman STEM Leadership Program, the Israel Science Foundation (grant No. 456/18), the Ministry of Science, Technology, and Space, Israel (grant No. 3-17426), the Tel Aviv University Center for Combatting Pandemics, and the Nicholas and Elizabeth Slezak Super Center for Cardiac Research and Biomedical Engineering at Tel Aviv University. R.J.A thanks the Israel Science Foundation (grant No. 1553/18) for the support of this research. G.S. thanks the Marian Gertner Institute for Medical Nanosystems in Tel Aviv University for their financial support.

\section{Author Contributions}

The manuscript was written through contributions of all authors. All authors have given approval to the final version of the manuscript. $\$$ These authors contributed equally.

\section{Additional Information}

Supplementary information including the detailed synthesis and characterization of the PEGdendrons, instrumentation and additional data are available.

\section{Table of Content}

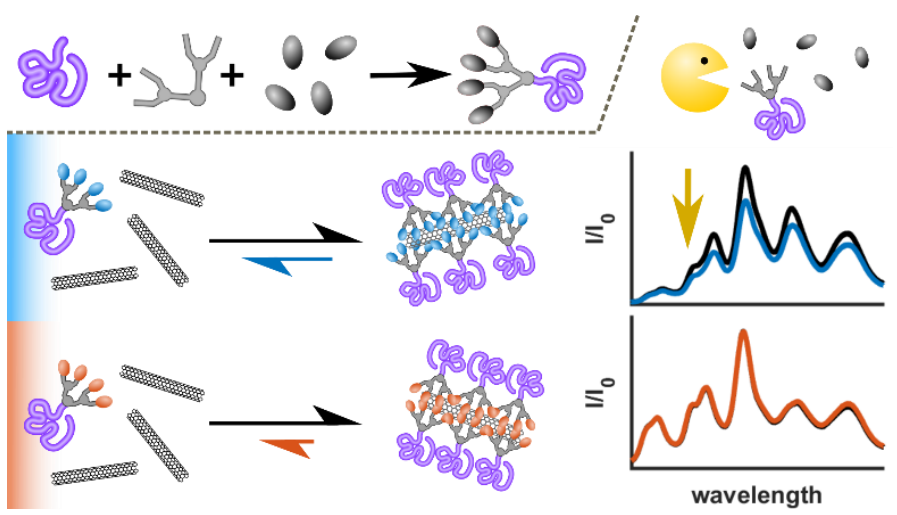

